The delightful essay by Mackay, of Birkbeck College, University of London, is well worth reading in its own right and, considering the role that he has played in the development of this subject, it is fitting that he should have the honour of writing the introductory chapter. With broad coverage and innumerable scholarly asides, Mackay succeeds in mentioning magic, determinism, chaos, religion, the date of Easter, Olber's paradox and the legal profession, as well as the Babylonians and the Greeks. His article also contains a charming example of the absence of cohesion between the contributors, characteristic of this kind of book: Mackay refers to the synthesis of the 'Platonic' molecule $(\mathrm{CH})_{20}$, as an 'Everest of alicyclic chemistry', hoping (p. 15) that it could be crystallized and its structure determined; Kuck, of Universität Bielefeld, in his article at the end of the book (p. 290), casually mentions this same molecule as 'of course ... well established ...', giving the reference to its structure determination, which was published in 1986!

School of Chemistry

J. H. ROBERTSON

University of Leeds

Leeds LS2 9JT

England

Acta Cryst. (1993). A49, 215

Introductory solid state physics. By H. P. MYERS. Pp $x i+546$. London: Taylor and Francis, 1990. Price (paper) £18.00. ISBN 0-85066-761-5.

This is an undergraduate textbook aimed at the second/third-year level. The author's stated objectives are to limit theoretical demands, use simple models, provide reasonable coverage and include some present-day research. These aims have been beautifully fulfilled in an eminently readable fashion. Several good points make this book better than average. There is a splendid introduction that explains the subject matter of solid-state physics and that impresses upon the reader the rewards of studying this subject. Chapter 1 is an unusual opening; it gives the reader a feeling for the magnitude of experimental fields, the phenomena found in the subject - such as superconductivity - and a tour around the Periodic Table. The next four chapters deal with properties other than electronic ones, including X-ray characterization, defects in solids, and phonons. There follow three chapters on the basics of electrons in materials, including free-electron theory, periodic potentials and cohesion. The origin of band gaps - a difficult concept - is dealt with cogently in Chapter 7 beginning with a simple Bragg reflected and transmitted plane wave. The author then explains Brillouin zones and other lattice effects before attempting a full solution for the nearly free electron model. The remainder of the chapter introduces band structure and the density of states in different materials. This is an important point since pictures of the density of states convey much insight. Metals, semiconductors, magnetic properties and dielectric properties each have a chapter. This forms the author's notion of a basic coverage. The specialist topics are: superconductivity, surface physics and the nucleus in solid-state physics particularly NMR.

Modern topics appear frequently throughout the book: the quantum Hall effect, organic semiconductors, high- $T$, superconductors and magnetic bubbles - to name a few. The subject matter is nicely dealt with, featuring many experimental results and lucid diagrams. Asides are put into special boxes and complicated mathematics are relegated to Appendices - in fact BCS theory is explained without equations! Each chapter has a list of references and most have problems. Some problems are not numerical, but are questions requiring a descriptive answer; only the numerical problems have answers at the back. I recommend this book and, as an additional endorsement, add that it is the textbook used at Leeds for our third-year solid-state physics course.

\section{Department of Physics}

University of Leeds

Leeds LS2 $9 \mathrm{JT}$

England

\section{B. J. HICKEY}

\section{Books Received}

Acta Cryst. (1993). A49, 215

The following books have been received by the Editor. Brief and generally uncritical notices are given of works of marginal crystallographic interest; occasionally, a book of fundamental interest is included under this heading because of difficulty in finding a suitable reviewer without great delay.

Salt effects in organic and organometallic chemistry. By A. LOUPY and B. TCHOUBAR. Pp. xiv +322 . Weinheim and New York: VCH Publishers, 1992. Price DM 164.00. ISBN 3-527-28025-1. A translation of the 1988 French edition, the book sets out to show the importance of the part played by salts in the development of chemical reactions in solution. 